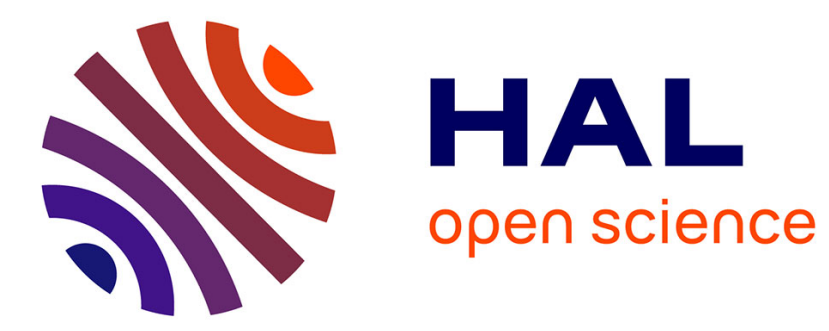

\title{
Preliminary in silico experiments: Towards new cancer treatments?
}

Michel Fliess, Cedric Join, Kaouther Moussa, Seddik Djouadi, Mohamed

Alsager

\section{- To cite this version:}

Michel Fliess, Cedric Join, Kaouther Moussa, Seddik Djouadi, Mohamed Alsager. Preliminary in silico experiments: Towards new cancer treatments?. 2021. hal-03191649

\section{HAL Id: hal-03191649 \\ https://hal-polytechnique.archives-ouvertes.fr/hal-03191649}

Preprint submitted on 11 Apr 2021

HAL is a multi-disciplinary open access archive for the deposit and dissemination of scientific research documents, whether they are published or not. The documents may come from teaching and research institutions in France or abroad, or from public or private research centers.
L'archive ouverte pluridisciplinaire HAL, est destinée au dépôt et à la diffusion de documents scientifiques de niveau recherche, publiés ou non, émanant des établissements d'enseignement et de recherche français ou étrangers, des laboratoires publics ou privés. 


\title{
Preliminary in silico experiments: Towards new cancer treatments?
}

\author{
Michel Fliess $^{1,5}$, Cédric Join ${ }^{2,5}$, Kaouther Moussa ${ }^{3}$, Seddik M. Djouadi ${ }^{4}$ and Mohamed Alsager ${ }^{4}$
}

\begin{abstract}
We present some "in silico" experiments to design combined chemo- and immunotherapy treatment schedules. We introduce a new framework by combining flatness-based control along with model-free control. The flatness property of the used mathematical model yields straightforward reference trajectories. They provide us with the nominal open-loop control inputs. Closing the loop via model-free control allows to deal with the uncertainties on the injected drug doses. Several numerical simulations illustrating different case studies are displayed. We show in particular that the considered health indicators are driven to the safe region, even for critical initial conditions. Furthermore, in some specific cases there is no need to inject chemotherapeutic agents.
\end{abstract}

Keywords: Cancer, chemotherapy, immunotherapy, flatness-based control, model-free control.

\section{INTRODUCTION}

Mathematical modeling in oncology is a well established discipline (see, e.g., [Altrock et al. (2015)] for recent advances). We consider the problem of drug injections scheduling from a control point of view. Among the many models which have been used, those stemming from the earlier work of [Stepanova (1979)] are quite popular. Most appealing are several publications by d'Onofrio and different coauthors: see especially [d'Onofrio et al. (2012)]. Such approaches to chemo- and immunotherapy led in recent years to promising control-theoretic investigations: see, e.g., [Alamir (2014)], [Schättler \& Ledzewicz (2015)] and references therein, [Moussa et al. (2020)], [Sharifi et al. (2017)],

[Sharifi et al. (2020a)]. They employ various optimization techniques which are related to optimal control, model predictive control, and robust control.

We explore here another route via tools which are combined for the first time and do not seem to have been used before in oncology, although they gave rise to an abundant literature in control engineering:

1) Flatness-based control (see [Fliess et al. (1995)], [Fliess et al. (1999)], and [Lévine (2009)],

[Sira-Ramírez \& Agrawal (2004)]) has been well received in many industrial domains. See, e.g.,

[Bonnabel \& Clayes (2020)] for tower cranes.

2) Besides being useful in concrete case-studies (see, e.g., [Amasyali et al. (2020)], [Park et al. (2021)],

[Park \& Olama (2021)] for energy management), model-free control in the sense of [Fliess \& Join (2013)] has already been illustrated by drug injections for some type-1 diabetes [MohammadRidha et al. (2018)] and for inflammation [Bara et al. (2018)]. Note that the terminology "model-free control" has been used many times with different definitions: see, e.g., [Chareyron \& Alamir (2009)] in oncology.

Our virtual patient is modeled through two ordinary differential equations presented in [d'Onofrio et al. (2012)]. This system is trivially flat with obvious flat outputs. The design of suitable reference trajectories with the corresponding openloop controls becomes straightforward. A major source of uncertainty, according to [Sharifi et al. (2020a)], is the unknown fluctuation of the drug delivery to the tumor, which should be related to actuators faults, i.e., to a classic topic in fault-tolerant control (see, e.g., [Noura et al. (2015)]). It has been already noticed that model-free control is well-suited for dealing with actuators faults: see [Fliess \& Join (2013)] for an academic example and [Lafont et al. (2015)] for a concrete case-study. The loop is therefore closed via model-free control. Let us emphasize the following points:

- the computer implementation is easy;

- only a low computing cost is necessary;

- some scenarios, i.e., in silico experiments, lead to unexpected results and should attract the attention of cancerologists.

Our paper is organized as follows. Section II reviews briefly modeling, flatness-based control, and model-free control. Numerical simulations are presented in Section III. Section IV contains some suggestions for future research.

\footnotetext{
${ }^{1}$ LIX (CNRS, UMR 7161), École polytechnique, 91128 Palaiseau, France (e-mail: Michel.Fliess@ polytechnique.edu)

2 CRAN (CNRS, UMR 7039)), Université de Lorraine, BP 239, 54506 Vandœuvre-lès-Nancy, France (e-mail: cedric.join@univ-lorraine.fr)

${ }^{3}$ Université Grenoble Alpes, CNRS, Grenoble INP, GIPSA-lab, 38000 Grenoble, France (e-mail: kaouther.moussa@ gipsa-lab.fr)

${ }^{4}$ Department of Electrical Engineering and Computer Science, University of Tennessee, Knoxville, TN 37996, USA (e-mail: mdjouadi@utk.edu, malsager@vols.utk.edu)

${ }^{5}$ AL.I.E.N., 7 rue Maurice Barrès, 54330 Vézelise, France (e-mail: \{michel.fliess, cedric.join\}@ @alien-sas.com)
} 


\section{Modeling AND CONTROL}

\section{A. Modeling for combined chemo- and immunotherapy}

We consider the model presented in [d'Onofrio et al. (2012)]

$$
\begin{aligned}
& \dot{x}=-\mu_{C} x \ln \left(\frac{x}{x_{\infty}}\right)-\gamma x y-x u \eta_{x} \\
& \dot{y}=\mu_{I}\left(x-\beta x^{2}\right) y-\delta y+\alpha+y v \eta_{y}
\end{aligned}
$$

$x, y$ are, respectively, the number of tumor cells and the immune cell density; the control variables $u$ and $v$ are the cytotoxic and immune-stimulation drugs; the parameters $\mu_{C}, \mu_{I}, \alpha, \gamma, \delta, x_{\infty}$ are positive. The terms $\eta_{x}, \eta_{y}, 0 \leq \eta_{x} \leq 1,0 \leq \eta_{y} \leq 1$, are inspired by [Sharifi et al. (2020a)]: they represent the uncertain and fluctuating parts of drugs which are delivered to the tumor.

\section{B. Flatness property}

A control system with $m$ independent control variables is said to be (differentially) flat if, and only if, there exists $m$ system variables $y_{1}, \ldots, y_{m}$, the flat outputs, such that any system variable $z$, the control variables for instance, may be expressed as a differential function of $y_{1}, \ldots, y_{m}$, i.e., $z=\Phi\left(y_{1}, \ldots, y_{m}, \ldots, y_{1}^{\left(\nu_{1}\right)}, \ldots, y_{m}^{\left(\nu_{m}\right)}\right)$, where the derivation orders $\nu_{1}, \ldots, \nu_{m}$ are finite. A linear system is flat if, and only if, it is controllable. Thus flatness may be viewed as another extension of Kalman's controllability.

Equations (1)-(2) yield

$$
\begin{aligned}
& u=\frac{\dot{x}+\mu_{C} x \ln \left(\frac{x}{x_{\infty}}\right)+\gamma x y}{-x \eta_{x}}=X(x, \dot{x}, y) \\
& v=\frac{\dot{y}-\mu_{I}\left(x-\beta x^{2}\right) y+\delta y-\alpha}{y \eta_{y}}=Y(y, \dot{y}, x)
\end{aligned}
$$

The above equations show immediately that System (1)-(2) is flat; $x, y$ are flat outputs.

\section{Reference trajectory and nominal open-loop control}

One of the main benefits of flatness is the possibility of easily deriving a suitable reference trajectory and the corresponding nominal open-loop control. For a given reference trajectory $x^{\star}(t), y^{\star}(t)$, the corresponding nominal control variables $u^{\star}(t)=$ $X\left(x^{\star}(t), \dot{x}^{\star}(t), y^{\star}(t)\right), v^{\star}(t)=Y\left(y^{\star}(t), \dot{y}^{\star}(t), x^{\star}(t)\right)$ might exhibit unacceptable negative values. Define therefore the nominal open-loop control variables $u_{\mathrm{OL}}(t)=u^{\star}(t)$ if $u^{\star}(t) \geq 0, u_{\mathrm{OL}}(t)=0$ if $u^{\star}(t)<0$, and $v_{\mathrm{OL}}(t)=v \star(t)$ if $v^{\star}(t) \geq 0, v_{\mathrm{OL}}(t)=0$ if $v^{\star}(t)<0$.

\section{Closing the loop via model-free control}

From a control-engineering standpoint the terms $\eta_{x}$ and $\eta_{y}$ should be related to actuators faults. Introduce therefore the two "decoupled" ultra-local models ([Fliess \& Join (2013)], [Lafont et al. (2015)]):

$$
\begin{aligned}
& \dot{z}_{x}=F_{x}+\alpha_{x} u_{\mathrm{MFC}} \\
& \dot{z}_{y}=F_{y}+\alpha_{y} v_{\mathrm{MFC}}
\end{aligned}
$$

where $z_{x}=x-x^{\star}, z_{y}=y-y^{\star}$ are the tracking errors; $\alpha_{x}$ (resp. $\alpha_{y}$ ) is a constant parameter which is chosen by the practitioner such that $\dot{x}$ and $\alpha_{x} u$ (resp. $\dot{y}$ and $\alpha_{y} v$ ) are of the same order of magnitude; $F_{x}$ and $F_{y}$, which are data-driven, subsume the poorly known structures and disturbances. A real-time estimation ([Fliess \& Join (2013)]) of $F_{x}, F_{y}$ are given by

$$
\begin{aligned}
& F_{x}^{\mathrm{est}}=-\frac{6}{\tau_{x}^{3}} \int_{t-\tau_{x}}^{t}\left((t-2 \sigma) x(\sigma)+\alpha_{x} \sigma\left(\tau_{x}-\sigma\right) u_{\mathrm{MFC}}(\sigma)\right) d \sigma \\
& F_{y}^{\mathrm{est}}=-\frac{6}{\tau_{y}^{3}} \int_{t-\tau_{y}}^{t}\left((t-2 \sigma) y(\sigma)+\alpha_{y} \sigma\left(\tau_{y}-\sigma\right) v_{\mathrm{MFC}}(\sigma)\right) d \sigma
\end{aligned}
$$

where $\tau_{x}, \tau_{y}>0$ are "small." Close the loop via an intelligent Proportional controller, or $i P$,

$$
\begin{aligned}
& u_{\mathrm{MFC}}=-\frac{F_{x}^{\mathrm{est}}+K_{x, P} z_{x}}{\alpha_{x}} \\
& v_{\mathrm{MFC}}=-\frac{F_{y}^{\mathrm{est}}+K_{y, P} z_{y}}{\alpha_{y}}
\end{aligned}
$$

where $K_{x, P}, K_{y, P}>0$. From

$$
\dot{z}_{x}+K_{x, P} z_{x}=0 \quad \dot{z}_{y}+K_{y, P} z_{y}=0
$$


it follows that those two gains ensure local stability around the reference trajectory.

The close-loop controls $u_{\mathrm{CL}}, v_{\mathrm{CL}}$ may now be defined:

- if $u_{\mathrm{OL}}+u_{\mathrm{MFC}} \geq 0$, then $u_{C L}=u_{\mathrm{OL}}+u_{\mathrm{MFC}}$;

- if $u_{\mathrm{OL}}+u_{\mathrm{MFC}}<0$, then $u_{\mathrm{CL}}=0$;

- if $v_{\mathrm{OL}}+v_{\mathrm{MFC}} \geq 0$, then $v_{C L}=v_{\mathrm{OL}}+v_{\mathrm{MFC}}$;

- if $v_{\mathrm{OL}}+v_{\mathrm{MFC}}<0$, then $v_{\mathrm{CL}}=0$.

\section{NUMERICAL SIMULATIONS}

\section{A. Presentation}

A huge number in silico experiments have been most easily performed. Only a few of them are presented below. Like in several other papers, the parameters in System (1)-(2) are given in Table 1 ([d'Onofrio et al. (2012)]). There are three equilibria corresponding to $\dot{x}=\dot{y}=u=v=0: 1$ ) a locally stable equilibrium $x=73, y=1.32$ which corresponds to a benign case; 2) an unstable saddle point $x=356.2, y=0.439$, which separates the benign and malignant regions; 3 ) a locally stable equilibrium $x=737.3, y=0.032$, which is malignant. The duration of an experiment is 60 days. The time sampling interval is equal to 30 minutes.

\section{B. Closed-loop and total amount of drugs}

Set $\eta_{x}=\eta_{y}=0.5$. This nominal value might be large according to [Sharifi et al. (2020a)]. Figures 1 and 2 display two experiments with the same initial point $x=500, y=0.5$, which lies in the attraction region of the malignant equilibrium. The total amounts of injected drugs, which are often considered as important constraints, are given by the two integrals $\int_{0}^{T} u_{\mathrm{CL}}(\tau) d \tau, \int_{0}^{T} v_{\mathrm{CL}}(\tau) d \tau$, where $T$ is the experiment duration. Figure 3 indicates that the quantity of drugs injected during the slow scenario is lower than in the fast one. This outcome ought to be discussed in oncology.

\section{Other scenarios}

1) Same initial point.: Here $\eta_{x}=0.25, \eta_{y}=0.75$ are supposed to be unknown. Use the same nominal parameters as in Section III-B, and the feedback loop of Section II-D, with $\alpha_{x}=-10000, \alpha_{y}=1, K_{x, P}=100, K_{y, P}=10$. The results depicted in Figures 4 and 5 show that the benign equilibrium is reached after a short period of time.

2) New initial point.: The virtual patient is in a critical state, i.e., the initial state $x=770, y=0.1$ is close to the malignant equilibrium. The time variation of $\eta_{x}$ and $\eta_{y}$, which are displayed in Figure 8 , are assumed to be unknown. It is possible to cure the virtual patient without the cytotoxic drug, i.e., $u_{\mathrm{CL}} \equiv 0$. Figure 6 , which should be of interest for cancerologists, exhibits a convergence to the benign equilibrium with some oscillations perhaps due to the violent fluctuations of $\eta_{y}$. The quality of the open loop behavior in Figure 7 is lower.

\section{CONCLUSION}

\section{A. Main goal}

According to our computer experiments, there are critical situations where only immunotherapy matters: the cytotoxic drugs are useless. Those startling calculations need of course to be further analyzed.

\section{B. Systems Biology}

In the spirit of Systems Biology (see, e.g., [Del Vecchio \& Murray (2015)]), let us suggest the the following research tracks:

- Examine the parameter identification in Equation (1).

- Lack of time prevented us for examining more thoroughly the unavoidable constraints especially for the cytotoxic and immune-stimulation drugs. It will be done in a future publication thanks to the optimization techniques associated to flatness-based control (see, e.g., [Petit et al. (2001)], [Sira-Ramírez \& Agrawal (2004)]).

- Flatness-based control might be helpful elsewhere:

1) Another model due to [Hahnfeldt et al. (1999)] has also been investigated from a control-theoretic perspective (see, e.g., [Kovács et al. (2014)], [Schättler \& Ledzewicz (2015)] and references therein, [Cacace et al. (2018)]). It is easy to check that it is flat.

2) The unicycle in [Sharifi et al. (2020b)], which is used as a nanorobot for drug delivery, is well known to be flat. 


\begin{tabular}{|c|c|c|}
\hline Parameter & Definition & Numerical Value \\
\hline$\mu_{C}$ & tumor growth rate & $1.0078 \cdot 10^{7} \mathrm{cells} / \mathrm{day}$ \\
$\mu_{I}$ & tumor stimulated proliferation rate & $.0029 \mathrm{day}^{-1}$ \\
$\alpha$ & rate of immune cells influx & $.0827 \mathrm{day}^{-1}$ \\
$\beta$ & inverse threshold & .0040 \\
$\gamma$ & interaction rate & $1 \cdot 10^{7} \mathrm{cells} /$ day \\
$\chi$ & death rate & $.1873 \mathrm{day}-1$ \\
$\sigma$ & chemotherapeutic killing parameter & $1 \cdot 10^{7} \mathrm{cells} / \mathrm{day}$ \\
$\lambda$ & immunotherapy injection parameter & $1 \cdot 10^{7}$ cells/day \\
$x_{\infty}$ & fixed carrying capacity & $780 \cdot 10^{6}$ cells \\
\hline
\end{tabular}

TABLE I:
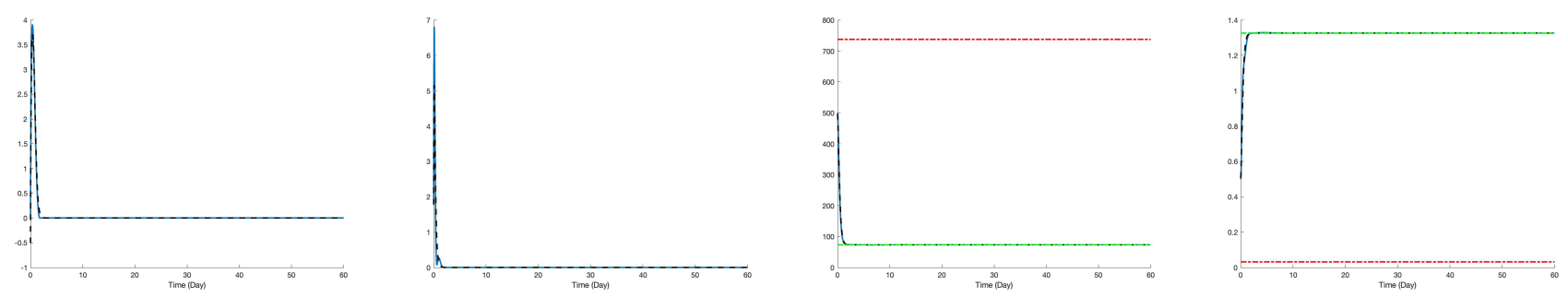

(a) Control $u$ (blue --) and $\operatorname{Nominal}(b) \operatorname{Control} v$ (blue --) and Nominal $(c)$ Output $x$ (blue - -), Reference( $d$ ) Output $y$ (blue --), Reference control $u^{*}$ (black --) control $v^{*}($ black --) trajectories (black --) and Stable trajectories (black --) and Stable points (red and green - .) points (red and green - .)

Fig. 1: Fast trajectory
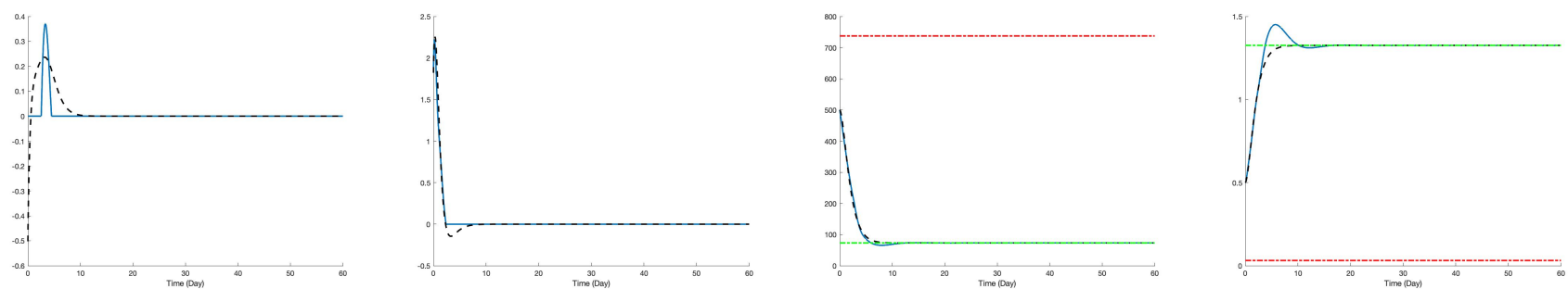

(a) Control $u$ (blue -) and $\operatorname{Nominal}(b)$ Control $v$ (blue - ) and Nominal $(c)$ Output $x$ (blue - ), Reference tra- $(d)$ Output $y$ (blue - ), Reference tracontrol $u^{*}($ black --$)$ control $v^{*}($ black --) jectories (black --) and Stable jectories (black - -) and Stable points (red and green -.) points (red and green -.)

Fig. 2: Slow trajectory
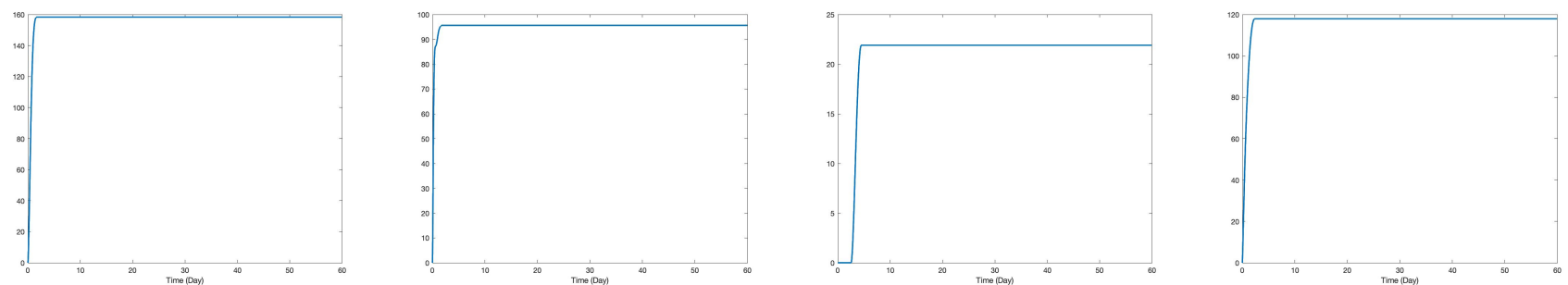

(a) Control $u$ integral of figure 1-(a) (b) Control $v$ integral of figure 1-(b) (c) Control $u$ integral of figure 2-(a) (d) Control $v$ integral of figure 2-(b)

Fig. 3: Comparison between total drug injections 

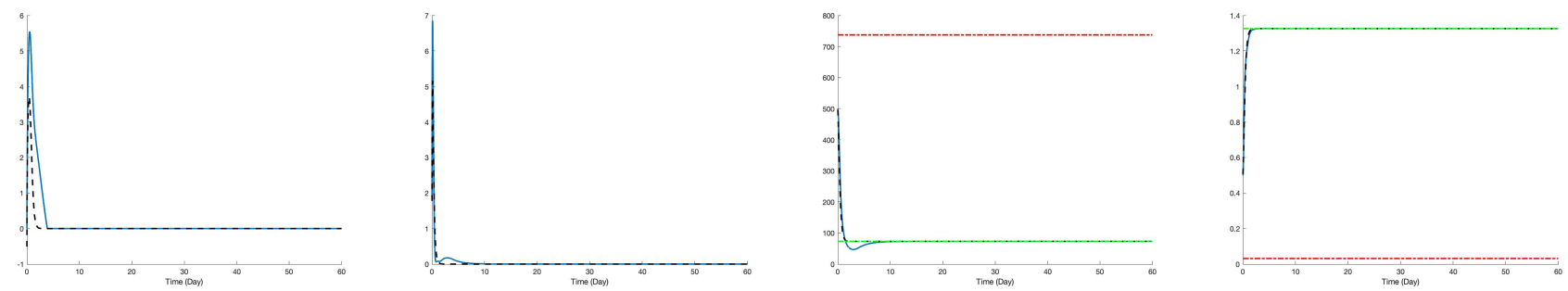

(a) Control $u$ (blue -) and $\operatorname{Nominal}(b)$ Control $v$ (blue -) and Nominal $(c)$ Output $x$ (blue - ), Reference tra- $(d)$ Output $y$ (blue -), Reference tracontrol $u^{*}($ black --) control $v^{*}($ black --)

Fig. 4: Unknown variation of $\eta_{x}$
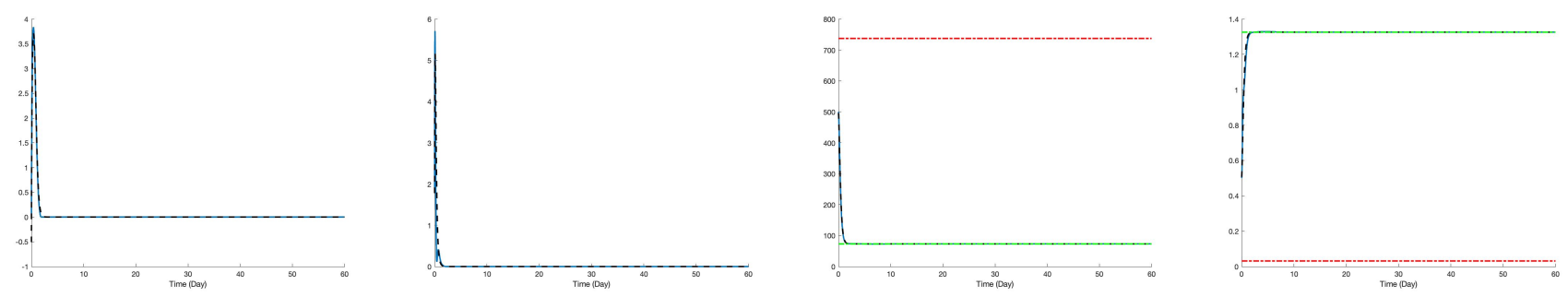

(a) Control $u$ (blue -) and $\operatorname{Nominal}(b)$ Control $v$ (blue -) and Nominal $(c)$ Output $x$ (blue - ), Reference tra- $(d)$ Output $y$ (blue -), Reference tracontrol $u^{*}($ black --) control $v^{*}$ (black --)
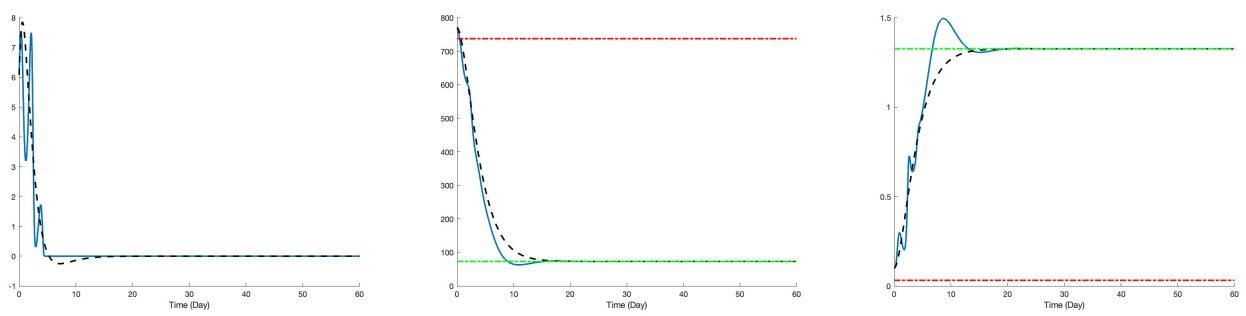

(a) Control $u$ (blue -) and Nominal $(b)$ Control $v$ (blue -) and Nominal $(c)$ Output $x$ (blue -), Reference tra- $(d)$ Output $y$ (blue -), Reference tracontrol $u^{*}($ black --) control $v^{*}$ (black --)
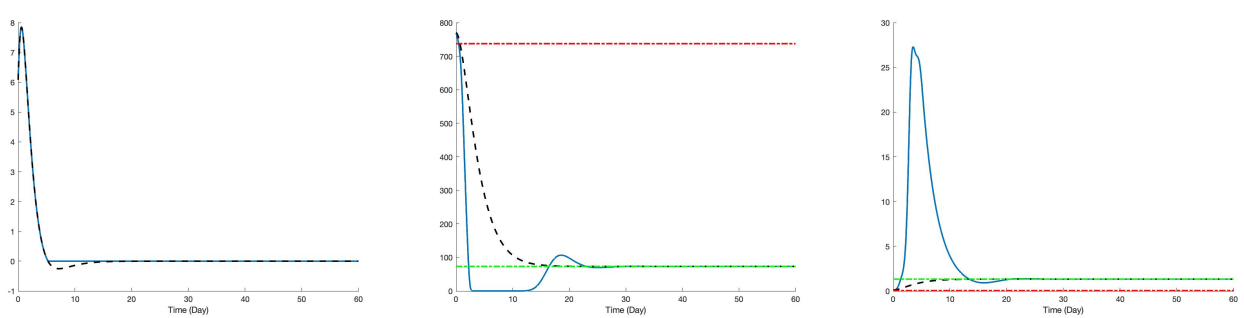

(a) Control $u$ (blue -) and $\operatorname{Nominal}(b)$ Control $v$ (blue -) and Nominal $(c)$ Output $x$ (blue -), Reference tra- $(d)$ Output $y$ (blue -), Reference tracontrol $u^{*}$ (black --)

control $v^{*}$ (black --) jectories (black --) and Stable points (red and green -.) jectories (black --) and Stable points (red and green -.)
Fig. 6: Very sick patient

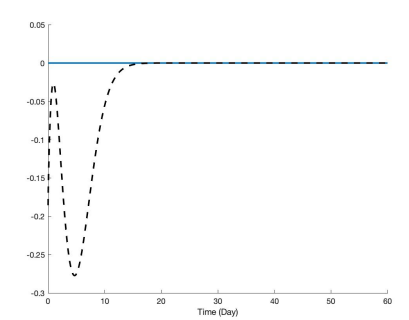
jectories (black --) and Stable jectories (black --) and Stable points (red and green -.) points (red and green -.)

Fig. 7: Open loop 
bioRxiv preprint doi: https://doi.org/10.1101/2021.04.06.438636; this version posted April 6, 2021. The copyright holder for this preprint (which was not certified by peer review) is the author/funder. All rights reserved. No reuse allowed without permission.

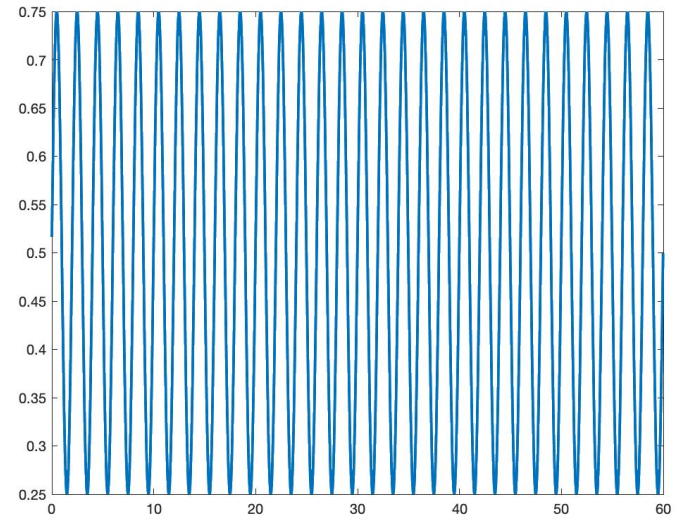

(a) Time evolution of $\eta_{x}$

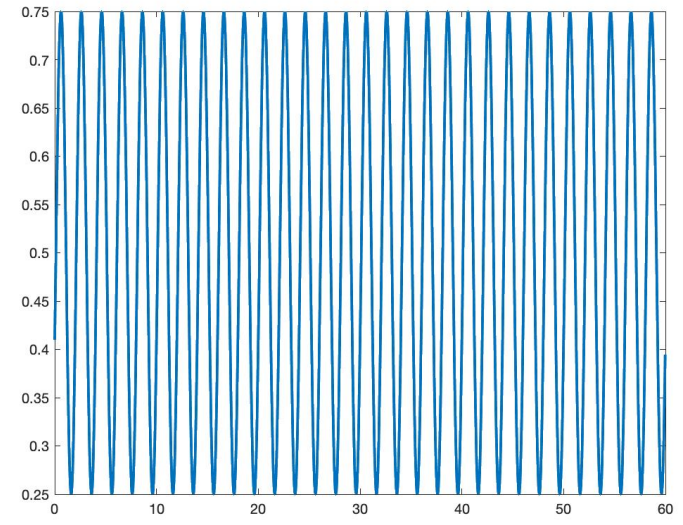

(b) Time evolution of $\eta_{y}$

Fig. 8: Fluctuation of the drug delivery 
bioRxiv preprint doi: https://doi.org/10.1101/2021.04.06.438636; this version posted April 6, 2021. The copyright holder for this preprint (which was not certified by peer review) is the author/funder. All rights reserved. No reuse allowed without permission.

\section{REFERENCES}

[Alamir (2014)] Alamir M. (2014). Robust feedback design for combined therapy of cancer. Optim. Control Appl. Meth., 35, 77-88.

[Altrock et al. (2015)] Altrock P.M., Liu L., Michor F. (2015). The mathematics of cancer: integrating quantitative models. Nature Rev. Cancer, 15 , 730-745.

[Amasyali et al. (2020)] Amasyali K., Chen Y., Telsang B., Olama M., Djouadi S.M. (2020). Hierarchical model-free transactional control of building loads to support grid services. IEEE Access, 8, 219367-219377.

[Bara et al. (2018)] Bara O., Fliess M., Join C., Day J., Djouadi S.M. (2018). Toward a model-free feedback control synthesis for treating acute inflammation. J. Theoret. Bio., 448, 26-37.

[Bonnabel \& Clayes (2020)] Bonnabel S., Clayes X. (2020). The industrial control of tower cranes: An operator-in-the-loop approach. IEEE Contr. Syst. Magaz., 40, 27-39.

[Cacace et al. (2018)] Cacace F., Cusimano V., Germani A., Palumbo A., Papa F. (2018). Closed-loop control of tumor growth by means of anti-angiogenic administration. Math. Biosci. Engin., 15, 827-839.

[Chareyron \& Alamir (2009)] Chareyron S., Alamir M. (2009). Model-free feedback design for a mixed cancer therapy. Biotech. Progr., 25, 690-700.

[Del Vecchio \& Murray (2015)] Del Vecchio M., Murray R.M. (2015). Biomolecular Feedback Systems. Princeton University Press.

[Fliess \& Join (2013)] Fliess M., Join C. (2013). Model-free control. Int. J. Contr., 86, 2228-2252.

[Fliess et al. (1995)] Fliess M., Lévine J., Martin P., Rouchon P. (1995). Flatness and defect of non-linear systems: introductory theory and examples. Int. J. Contr., 61, 1327-1361.

[Fliess et al. (1999)] Fliess M., Lévine J., Martin P., Rouchon P. (1999). A Lie-Bäcklund approach to equivalence and flatness of nonlinear systems. IEEE Trans. Automat. Contr., 44, 922-937.

[Hahnfeldt et al. (1999)] Hahnfeldt P., Panigrahy D., Folkman J., Hlatky L. (1999). Tumor development under angiogenic signaling: A dynamical theory of tumor growth, treatment response, and postvascular dormancy. Cancer Res., 59, 4770-4775.

[Kovács et al. (2014)] Kovács L., Szeles A., Sápi J., Drexler D.A., Rudas I., Harmati I., Sápi Z. (2014). Model-based angiogenic inhibition of tumor growth using modern robust control method. Comput. Meth. Program. Biomed., 114, e98-e110.

[Lafont et al. (2015)] Lafont F., Balmat J.-F., Pessel N., Fliess M. (2015). A model-free control strategy for an experimental greenhouse with an application to fault accommodation. Comput. Electron. Agricult., 110, 139-149.

[Lévine (2009)] Lévine J. (2009). Analysis and Control of Nonlinear Systems: A Flatness-based Approach. Springer.

[MohammadRidha et al. (2018)] MohammadRidha T., Ait-Ahmed M., Chaillous L., Krempf M., Guilhem I., Poirier J.Y., Moog C.H. (2018). Model free iPID control for glycemia regulation of type-1 diabetes. IEEE Trans. Biomed. Eng., 65, 199-206.

[Moussa et al. (2020)] Moussa K., Fiacchini M., Alamir M. (2020). Robust optimal scheduling of combined chemo- and immunotherapy: Considerations on chemotherapy detrimental effects. Amer. Contr. Conf., Denver.

[Noura et al. (2015)] Noura H., Theilliol D., Ponsart J.-C., Chamseddine A. (2009). Fault-tolerant Control Systems: Design and Practical Applications. Springer.

[d'Onofrio et al. (2012)] d'Onofrio A., Ledzewicz U., Schättler H. (2012). On the dynamics of tumor-immune system interactions and combined chemoand immunotherapy. A. d'Onofrio, P. Cerrai, A. Gandolfi (Eds.): New Challenges for Cancer Systems Biomedicine, Springer, pp. $249-266$.

[Park et al. (2021)] Park B., Zhang Y., Olama M., Kuruganti T. (2021). Model-free control for frequency response support in microgrids utilizing wind turbines. Elec. Power Syst. Res., 194, 107080.

[Park \& Olama (2021)] Park B., Olama M. (2021). A model-free voltage control approach to mitigate motor stalling and FIDVR for smart grids. IEEE Trans. Smart Grids, 12, 67-78.

[Petit et al. (2001)] Petit N., Milam M.B., Murray R.M. (2001). Inversion based constrained trajectory optimization. IFAC Proc. Vol., $34,1211-1216$.

[Schättler \& Ledzewicz (2015)] Schättler H., Ledzewicz U. (2015). Optimal Control for Mathematical Models of Cancer: An Application of Geometric Methods Springer, 2015.

[Sharifi et al. (2017)] Sharifi N., Ozgoli S., Ramezani A. (2017). Multiple model predictive control for optimal drug administration of mixed immunotherapy and chemotherapy of tumours. Comput. Meth. Program. Biomed., 144, 13-19.

[Sharifi et al. (2020a)] Sharifi N., Zhou Y., Holmes G., Chen Y. (2020a). Overcoming channel uncertainties in touchable molecular communication for direct-drug-targeting-assisted immuno-chemotherapy. IEEE Trans. Nanobiosci., 19, 249-258.

[Sharifi et al. (2020b)] Sharifi N., Ali M., Holmes G., Chen Y. (2020b). Blind Obstacle Avoidance Using Taxicab Geometry for NanorobotAssisted Direct Drug Targeting. 42nd Int. Conf. IEEE Engin. Med. Biol. Soc., Montreal.

[Sira-Ramírez \& Agrawal (2004)] Sira-Ramírez H., \& Agrawal S.K. (2004). Differentially Flat Systems. Marcel Dekker.

[Stepanova (1979)] Stepanova N.V. (1979). Course of the immune reaction during the development of a malignant tumour (in Russian). Biophysics, 24, 917-923. 